\title{
NONCONSTANT ENDOMORPHISMS OF LATTICES
}

\author{
J. SICHLER ${ }^{1}$
}

\begin{abstract}
There is a proper class of pairwise nonisomorphic lattices whose monoids of all nonconstant endomorphisms are isomorphic to a given monoid $\boldsymbol{M}$.
\end{abstract}

1. Introduction. There are monoids not appearing as full endomorphism monoids of lattices: every constant mapping of a lattice into itself is one of its endomorphisms and therefore any monoid of all endomorphisms of a lattice contains a left zero element. The nonconstant endomorphisms do not have to form a monoid since the composition of two nonconstant mappings may be a constant mapping.

The aim of the present note is to show that for every monoid $\boldsymbol{M}$ there is a lattice $L$ such that the set of all its nonconstant endomorphisms is closed under composition and isomorphic to $\boldsymbol{M}$; this solves completely Problem 3 of [2]. R. McKenzie advised the author that the result follows from [3] under the generalized continuum hypothesis. However, neither the present paper nor any of the results used here requires any set-theoretical assumptions.

Using graph-theoretic and lattice-theoretic results we will prove a substantially stronger theorem which will also yield the number of nonisomorphic lattices with isomorphic monoids of nonconstant endomorphisms.

2. Graphs. By a graph we will always mean a pair $\langle X, R\rangle$ in which $X$ is a set and $R$ is a set of two-element subsets of $X$; a compatible mapping $f:\langle X, R\rangle \rightarrow\left\langle X^{\prime}, R^{\prime}\right\rangle$ is a mapping $f: X \rightarrow X^{\prime}$ for which $\left\{x_{1}, x_{2}\right\} \in R$ implies $\left\{f\left(x_{1}\right), f\left(x_{2}\right)\right\} \in R^{\prime}$. Let $G$ be the category of all graphs and all their compatible mappings and let $\boldsymbol{H}$ be the full subcategory of $\boldsymbol{G}$ determined by all Hell graphs, i.e. by the graphs $\langle X, R\rangle$ satisfying the following condition:

For every $x$ in $X$ there is a three-element

$$
\begin{aligned}
& \text { set }\left\{x, x_{1}, x_{2}\right\} \subset X \text { such that }\left\{x, x_{1}\right\}, \\
& \left\{x, x_{2}\right\},\left\{x_{1}, x_{2}\right\} \in R .
\end{aligned}
$$

Presented to the Society, September 21, 1970; received by the editors April 8, 1971. AMS 1970 subject classifications. Primary 06A20, 18B15.

Key words and phrases. Lattice, homomorphism, category.

${ }^{1}$ This research work was supported by the National Research Council of Canada.

(c) American Mathematical Society 1972 
In other words, every vertex belongs to a triangle of the graph $\langle X, R\rangle$.

The following theorem is due to P. Hell [6].

THEOREM 1. Every full category of algebras is isomorphic to a full subcategory of $\boldsymbol{H}$, i.e. $\boldsymbol{H}$ is binding.

As every monoid $\boldsymbol{M}$ is isomorphic to the full endomorphism monoid of an algebra, the above theorem yields the existence of a Hell graph $H$ with the monoid of all compatible mappings $f: H \rightarrow H$ isomorphic to $\boldsymbol{M}$.

3. Lattices. Let $\boldsymbol{L}$ be the category of all lattices and all their homomorphisms and let $\boldsymbol{N}$ be the class of all lattices and all nonconstant homomorphisms between them; note that $N$ is not a category.

THEOREM 2. There is a full subcategory $\boldsymbol{M}$ of the category $\boldsymbol{L}$ of all lattices such that

(i) $M \cap N$ is a category,

(ii) $\boldsymbol{M} \cap \boldsymbol{N}$ is isomorphic to $\boldsymbol{H}$.

In other words, $\boldsymbol{M} \cap \boldsymbol{N}$ is a binding category. To prove the theorem a one-to-one and full functor $M: \boldsymbol{H} \rightarrow \boldsymbol{N}$ will be constructed; the objects of $\boldsymbol{M}$ will be all lattices $M(G)$ for $G=\langle X, R\rangle$ in $\boldsymbol{H}$. To define $M(G)$, consider the lattice $F(X)$ freely generated by $X$ and define $\Theta_{R}$ to be the smallest congruence relation on $F(X)$ identifying $x \wedge y$ with $z \wedge t$ and $x \vee y$ with $z \vee t$ whenever both $\{x, y\}$ and $\{z, t\}$ belong to $R$. Set $M(G)=F(X) / \Theta_{R}$. Let $\pi_{G}: F(X) \rightarrow M(G)$ be the canonical homomorphism with $\operatorname{Ker} \pi_{G}=\Theta_{R}$, let $f: G \rightarrow G^{\prime}=\left\langle X^{\prime}, R^{\prime}\right\rangle$ be a compatible mapping and let $F(f): F(X) \rightarrow F\left(X^{\prime}\right)$ be the homomorphism of the free lattices extending $f$. If $\left\{x_{1}, x_{2}\right\} \in R$, then there is $x_{3}$ in $X, x_{3} \neq x_{2}$, such that $\left\{x_{1}, x_{3}\right\} \in R$. Hence $x_{1} \vee x_{2} \equiv x_{1} \vee x_{3}\left(\Theta_{R}\right)$ and $x_{1} \wedge x_{2} \equiv x_{1} \wedge x_{3}\left(\Theta_{R}\right)$. As $f$ is a compatible mapping, then $F(f)\left(x_{1} \vee x_{2}\right)=$ $F(f)\left(x_{1}\right) \vee F(f)\left(x_{2}\right)=f\left(x_{1}\right) \vee f\left(x_{2}\right) \equiv f\left(x_{1}\right) \vee f\left(x_{3}\right)=F(f)\left(x_{1} \vee x_{3}\right)$ in $\Theta_{R^{\prime}}$. Similarly, $F(f)\left(x_{1} \wedge x_{2}\right) \equiv F(f)\left(x_{1} \wedge x_{3}\right)\left(\Theta_{R^{\prime}}\right)$. Consequently, $\Theta_{R} \subseteq \operatorname{Ker}\left(\pi_{G^{\prime}} \circ F(f)\right)$ so there is a unique homomorphism

$$
M(f): M(G) \rightarrow M\left(G^{\prime}\right)
$$

such that $M(f) \circ \pi_{G}=\pi_{G^{\prime}} \circ F(f)$. It is easy to see that $M$ is a functor from $\boldsymbol{H}$ into the category $\boldsymbol{L}$ of all lattices. Denote $e=\pi_{G}(x \vee y)=\pi_{G}(\bar{x} \vee \bar{y})$ for any $\{x, y\},\{\bar{x}, \bar{y}\}$ in $R$; similarly, $z=\pi_{G}(x \wedge y)$. Since the graphs satisfying $(\mathrm{H})$ do not have isolated points, $z \leqq \pi_{G}(x) \leqq e$ for every $x$ in $X$; as $M(G)$ is generated by $\pi_{G}(X), z \leqq m \leqq e$ for every $m$ in $M(G)$. Note also, that for $\left\{x_{1}, x_{2}\right\}$ in $R,\left\{\pi_{G}\left(x_{1}\right), \pi_{G}\left(x_{2}\right)\right\}$ is a complemented pair of elements of $M(G)$, so that, for every compatible $f: G \rightarrow G^{\prime}, M(f)(e)=e^{\prime}$ and $M(f)(z)=z^{\prime}$.

The following lemma is an easy consequence of Theorem 2 of [3]. 
Lemma 1. (1) If $m \in M(G) \backslash\{e, z\}$, then $\left|\pi_{G}^{-1}(m)\right|=1$.

(2) $\left\{m_{1}, m_{2}\right\}$ is a complemented pair of elements of $M(G)$ if and only if either $\left\{m_{1}, m_{2}\right\}=\{e, z\}$ or $\left\{m_{1}, m_{2}\right\}=\left\{\pi_{G}\left(x_{1}\right), \pi_{G}\left(x_{2}\right)\right\}$ for some $\left\{x_{1}, x_{2}\right\}$ in $R$.

Lemma 2. (3) The functor $M$ is one-to-one, $M: H \rightarrow N$.

(4) If $\varphi: M(G) \rightarrow M\left(G^{\prime}\right)$ is a lattice homomorphism such that $\varphi(e)=e^{\prime}$ and $\varphi(z)=z^{\prime}$, then $\varphi=M(f)$ for a compatible mapping $f: G \rightarrow G^{\prime}$.

Proof. (3) follows immediately from (1) and (H).

(4) $\varphi$ preserves all complemented pairs. Since every vertex $x$ of $\langle X, R\rangle$ belongs to a triangle of $R, \varphi(x) \in M\left(G^{\prime}\right) \backslash\left\{e^{\prime}, z^{\prime}\right\}$ by (2). The only other elements of $M\left(G^{\prime}\right)$ possessing complements are elements of the form $\pi_{G^{\prime}}(y)$ for $y$ in $X^{\prime}$ so that $\varphi\left(\pi_{G}(X)\right) \subseteq \pi_{G^{\prime}}\left(X^{\prime}\right)$. Using (1) and (2) again, we conclude that there is a compatible mapping $f: G \rightarrow G^{\prime}$ with $M(f) \circ \pi_{G}=$ $\pi_{G^{\prime}} \circ F(f)=\varphi \circ \pi_{G}$. Since $\pi_{G}$ is an onto homomorphism, $\varphi=M(f)$.

To finish the proof of the theorem it remains to show that all the other homomorphisms $\varphi: M(G) \rightarrow M\left(G^{\prime}\right)$ are constant.

If $\varphi(z)=e^{\prime}$ or $\varphi(e)=z^{\prime}$, then $\varphi$ is constant, as $z \leqq m \leqq e$ for all elements $m$ of $M(G)$, and therefore we may assume that $d=\varphi(z) \neq e^{\prime}, z^{\prime}$. For any triangle $\{a, b, c\}$ of $G, A=\left\{\pi_{G}(a), \pi_{G}(b), \pi_{G}(c), e, z\right\}$ is a simple sublattice of $M(G)$; set $\pi_{G}(s)=\bar{s}$ for every $s$ in $F(X)$. Consider the sublattice $\varphi(A)$ of $M\left(G^{\prime}\right)$. If $\varphi$ is not constant, then $\varphi \mid A$ has to be a one-to-one homomorphism. If $\varphi(\bar{a})=z^{\prime}$, then $\varphi(z) \leqq \varphi(\bar{a})=z^{\prime}$, a contradiction. $\varphi(\bar{a})=e^{\prime}$ implies $\varphi(\bar{b})=e^{\prime} \wedge \varphi(\bar{b})=\varphi(\bar{a} \wedge \bar{b})=\varphi(z)$, a contradiction again. We may assume that $\varphi(\bar{a}), \varphi(\bar{b}), \varphi(\bar{c}) \notin\left\{e^{\prime}, z^{\prime}\right\}$; since $d=\varphi(\bar{a}) \wedge \varphi(\bar{b})=\varphi(\bar{a}) \wedge \varphi(\bar{c})$, using (1) we obtain the equation

$$
\pi_{G^{\prime}}^{-1}(d)=\pi_{G^{\prime}}^{-1}(\varphi(\bar{a})) \wedge \pi_{G^{\prime}}^{-1}(\varphi(\bar{b}))=\pi_{G^{\prime}}^{-1}(\varphi(\bar{a})) \wedge \pi_{G^{\prime}}^{-1}(\varphi(\bar{c}))
$$

in the free lattice $F\left(X^{\prime}\right)$. By Lemma 2.6 of [6],

$$
\pi_{G^{\prime}}^{-1}(d)=\pi_{G^{\prime}}^{-1}(\varphi(\bar{a})) \wedge\left(\pi_{G^{\prime}}^{-1}(\varphi(\bar{b})) \vee \pi_{G^{\prime}}^{-1}(\varphi(\bar{c}))\right)
$$

in $F\left(X^{\prime}\right)$; consequently,

$$
d=\varphi(\bar{a}) \wedge(\varphi(\bar{b}) \vee \varphi(\bar{c}))=\varphi(\bar{a} \wedge(\bar{b} \vee \bar{c})) \text { in } M\left(G^{\prime}\right) .
$$

But $\bar{b} \vee \bar{c}=\bar{a} \vee \bar{c} \geqq \bar{a}$, therefore $\varphi(z)=d=\varphi(\bar{a}) ; \varphi \mid A$ cannot be one-to-one, thus $\varphi$ is a constant homomorphism of $M(G)$ into $M\left(G^{\prime}\right)$.

4. Number of representations. Note that $|M(X, R)|=|X|$ for any infinite $X$. Utilizing this together with the existence of a full embedding of the category of commutative groupoids into the category $\boldsymbol{H}$ by a functor $K$ preserving the infinite cardinalities of underlying sets (the existence of such a functor $K$ follows easily from [4] and [6]), we obtain the following theorem as an immediate consequence of Theorem 5 of [5]. 
THEOREM 3. Let $\boldsymbol{M}$ be a monoid, $|\boldsymbol{M}|=m$ and let $n$ be an infinite cardinal number, $n \geqq m$. Then there are exactly $2^{n}$ lattices $L_{\alpha}$ such that

(a) the set of all nonconstant endomorphisms of each $L_{\alpha}$ is isomorphic to $M$,

(b) $\left|L_{\alpha}\right|=n$ for each $\alpha \in 2^{n}$,

(c) given indices $\alpha, \alpha^{\prime} \in 2^{n}, \alpha \neq \alpha^{\prime}, \operatorname{Hom}_{L}\left(L_{\alpha}, L_{\alpha^{\prime}}\right)$ consists exactly of all constant homomorphisms.

COROLlARY. For every infinite cardinal number $n$ there is a lattice $L$ of cardinality $n$ such that its endomorphisms are constant mappings only.

\section{REFERENCES}

1. C. C. Chen and G. Grätzer, On the construction of complemented lattices, J. Algebra 11 (1969), 56-63. MR 38 \#1038.

2. G. Grätzer, Lattice theory: First concepts and distributive lattices, Freeman, San Francisco, Calif., 1971.

3. G. Grätzer and J. Sichler, On the endomorphism semigroup and category of bounded lattices, Pacific J. Math. 35 (1970), 639-647.

4. Z. Hedrlín and A. Pultr, On full embeddings of categories of algebras, Illinois J. Math. 10 (1966), 392-406. MR 33 \#85.

5. Z. Hedrlín and J. Sichler, Any boundable binding category contains a proper class of mutually disjoint copies of itself, Algebra Universalis 1 (1971), 97-103.

6. P. Hell, Full embeddings into some categories of graphs, Algebra Universalis (to appear).

7. B. Jónsson, Sublattices of a free lattice, Canad. J. Math. 13 (1961), 256-264 MR 23 \#A818.

Department of Mathematics, University of Manitoba, Winnipeg, Manitoba, Canada 